\title{
A SIMPLE CONSTRUCTIVE PROOF OF AN ANALOGUE OF THE CORONA THEOREM
}

\author{
MICHAEL VON RENTELN
}

\begin{abstract}
We present a simple and constructive proof (i.e. a proof without using Gelfand theory) of an analogue of the Corona theorem for the Wiener algebras $W$ and $\mathrm{W}^{+}$of absolutely convergent Fourier and Taylor series respectively, also the disc algebra $A(\bar{D})$ and the subalgebras $A^{n}(\bar{D})$ of functions whose $n$th derivatives extend continuously to $\bar{D}=\{z:|z|<1\}$.
\end{abstract}

Let $D$ denote the open unit disc in the complex plane, $T$ its boundary and $\bar{D}$ its closure. We consider the following algebras.

(i) The Wiener algebra

$$
W=\left\{f(z)=\sum_{-\infty}^{+\infty} a_{n} z^{n}: \sum_{-\infty}^{+\infty}\left|a_{n}\right|<\infty\right\}, \quad z \in T,
$$

of absolutely convergent Fourier series with the norm $\|f\|=\Sigma_{-\infty}^{+\infty}\left|a_{n}\right|$.

(ii) The subalgebra

$$
W^{+}=\left\{f(z)=\sum_{0}^{\infty} a_{n} z^{n}: \sum_{0}^{\infty}\left|a_{n}\right|<\infty\right\}, \quad z \in \bar{D},
$$

of absolutely convergent Taylor series with the norm $\|f\|=\Sigma_{0}^{\infty}\left|a_{n}\right|$.

(iii) The disc algebra

$$
A(\bar{D})=\{f: D \rightarrow \mathrm{C}: f \text { is analytic on } D \text { and extends continuously to } \bar{D}\}
$$

with the norm $\|f\|_{\infty}=\sup \{|f(z)|: z \in D\}$.

(iv) The subalgebras

$$
A^{n}(\bar{D})=\left\{f \in A(\bar{D}): f^{(n)} \text { extends continuously to } \bar{D}\right\},
$$

$n \in \mathbf{N}$, with the norm $\|f\|_{n}=\max \left\{\left\|f^{(k)}\right\|_{\infty}: 0 \leqslant k \leqslant n\right\}$, where $f^{(0)}=f$.

The algebras (i)-(iv) are complex commutative Banach algebras (with the usual pointwise algebraic operations and the norms given above) having an identity element. Therefore one can apply Gelfand theory to produce an elegant (but nonconstructive) proof of the following theorem, which is an analogue of the Corona theorem.

THEOREM. Let I be the ideal generated by the functions $f_{1}, \ldots, f_{N} \in A$, where $A$ is one of the Banach algebras above. Then $I=A$ if and only if the functions $f_{1}, \ldots, f_{N}$ have no common zero in $\bar{D}$ (or $T$ if $A=W$ ).

Received by the editors September 27, 1979.

AMS (MOS) subject classifications (1970). Primary 30A98; Secondary 46J15. 
The aim of this note is to present a constructive proof, i.e. a proof without using Gelfand's theory of maximal ideals, Zorn's Lemma, etc., and which is more general and considerably simpler than the existing constructive proofs in the literature. Also our proof is self-contained. In the case of the disc algebra $A(\bar{D})$ there are two different proofs given by L. Carleson [1] and P. J. Cohen [2]. Neither of them is particularly simple. Carleson's proof is based on the representation of the continuous linear functionals on $A(\bar{D})$ and on the theorem of F. and M. Riesz on analytic measures, whereas Cohen's proof is a careful and detailed translation of the abstract proof. In the cases of the Wiener algebras $W$ and $W^{+}$we know of constructive proofs only when $N=1$. A simple proof for $W$ was given by $\mathrm{D}$. $\mathrm{J}$. Newman [5] and, recently, for $W^{+}$by J. Spilker [6]. We note that the case $N=1$ is the original theorem of Wiener on the inversion of absolutely convergent Fourier (Taylor) series.

We will now give a proof of the theorem for the Wiener algebra $W^{+}$. The corresponding proofs for the other algebras differ only slightly by being somewhat simpler. In a remark at the end of this note we indicate the differences.

The idea behind the proof is quite simple and natural. We want to produce an element in the ideal $I$ which is invertible. In the case of the algebra $W$ one may take simply $\sum_{i=1}^{N} \bar{f}_{i} f_{i}$, because the complex conjugates $\bar{f}_{i}$ of the functions $f_{i} \in W$ belong also to $W$. For the other algebras $A$ this is not true. Therefore we shall try to find for the $\bar{f}_{i}$ substitutes $Q_{i}$ with $Q_{i} \in A$. This can be done in such a way that the resulting function $\sum_{i=1}^{N} Q_{i} f_{i}$ is almost invertible, i.e. after dividing by a finite Blaschke product the function is invertible in the algebra $A$ without leaving the ideal $I$.

Proof of THe Theorem. Let $A=W^{+}$. We note that $|f(z)|<\|f\|$ for $f \in W^{+}$ and $z \in \bar{D}$. Since the condition is obviously necessary we assume that $f_{1}, \ldots, f_{N}$ have no common zero in $\bar{D}$. Then

$$
\sum_{i=1}^{N}\left|f_{i}(z)\right|^{2} \geqslant 2 \delta \quad(z \in \bar{D})
$$

holds for some constant $\delta>0$. Let $C=\Sigma_{i=1}^{N}\left\|f_{i}\right\|_{\infty}$. Since the ideal $I$ has a finite number of generators

$$
f_{i}(z)=\sum_{n=0}^{\infty} a_{n i} z^{n} \quad(i=1, \ldots, N)
$$

there exists a positive integer $K$ such that

$$
\sum_{n>K}\left|a_{n i}\right|<\delta / C \text { for every } i=1, \ldots, N \text {. }
$$

Let $P_{i}$ be the $K$ th partial sum of $f_{i}$, i.e.

$$
P_{i}(z)=\sum_{n=0}^{K} a_{n i} z^{n}
$$

For the complex conjugates $\bar{f}_{i}, \bar{P}_{i}$ of the functions $f_{i}, P_{i}$ we estimate

$$
\left|\left(\bar{f}_{i}-\bar{P}_{i}\right)(z)\right|=\left|\left(f_{i}-P_{i}\right)(z)\right|<\left\|f_{i}-P_{i}\right\|=\sum_{n>K}\left|a_{n i}\right|<\delta / C
$$


for $z \in \bar{D}$ and all $i=1, \ldots, N$. After multiplication with $f_{i}$ and summation we obtain

$$
\sum_{i=1}^{N}\left|\left(\bar{f}_{i} f_{i}-\bar{P}_{i} f_{i}\right)(z)\right|<\frac{\delta}{C} \sum_{i=1}^{N}\left|f_{i}(z)\right|<\delta \quad(z \in \bar{D}) .
$$

From this it follows that

$$
\sum_{i=1}^{N}\left|f_{i}(z)\right|^{2}-\left|\sum_{i=1}^{N}\left(\bar{P}_{i} f_{i}\right)(z)\right|<\delta \quad(z \in \bar{D}) .
$$

Using (1) we obtain

$$
\left|\sum_{i=1}^{N}\left(\bar{P}_{i} f_{i}\right)(z)\right| \geqslant \delta \quad(z \in \bar{D}) .
$$

Let us define functions

$$
Q_{i}(z)=\sum_{n=0}^{K} \bar{a}_{n i} z^{K-n} \quad(i=1, \ldots, N) .
$$

We note that the $Q_{i}$ are polynomials in $z$, in particular, $Q_{i} \in W^{+}$. For $z \in T$ we have $\bar{z}=1 / z$ and therefore $Q_{i}(z)=z^{K} \bar{P}_{i}(z)$. From (2) it follows that

$$
\left|\sum_{i=1}^{N}\left(Q_{i} f_{i}\right)(z)\right|>\delta \quad(z \in T) .
$$

The function $f=\sum_{i=1}^{N} Q_{i} f_{i}$ is an element of the ideal $I$ and (3) implies that $f$ has no zero on $T$. Therefore $f(z)=\sum_{n=0}^{\infty} a_{n} z^{n}$ has only a finite number of zeros in $D$. Let $z_{0}$ be such a zero. We first show that

$$
g(z)=\frac{f(z)}{z-z_{0}}=\sum_{n=0}^{\infty} c_{n} z^{n} \in W^{+} .
$$

A short computation yields $c_{n}=P_{n}\left(z_{0}\right) /-z_{0}^{n+1}$, where $P_{n}(z)$ is the $n$th partial sum of $f$. Since $f\left(z_{0}\right)=0$ we obtain $P_{n}\left(z_{0}\right)=-\left(a_{n+1} z_{0}^{n+1}+a_{n+2} z_{0}^{n+2}+\ldots\right)$; thus

$$
c_{n}=a_{n+1}+a_{n+2} z_{0}+a_{n+3} z_{0}^{2}+\ldots
$$

For the first coefficients we get

$$
\begin{aligned}
& \left|c_{0}\right| \leqslant\left|a_{1}\right|+\left|a_{2}\right|\left|z_{0}\right|+\left|a_{3}\right|\left|z_{0}\right|^{2}+\ldots \\
& \left|c_{1}\right| \leqslant\left|a_{2}\right|+\left|a_{3}\right|\left|z_{0}\right|+\left|a_{4}\right|\left|z_{0}\right|^{2}+\ldots \\
& \left|c_{2}\right| \leqslant\left|a_{3}\right|+\left|a_{4}\right|\left|z_{0}\right|+\left|a_{5}\right|\left|z_{0}\right|^{2}+\ldots
\end{aligned}
$$

Since the series occurring in the rows and columns are absolutely convergent (because $f \in W^{+}$and $\left|z_{0}\right|<1$ ) we can take the sum for each column. Observing that

$$
\left|a_{n}\right|+\left|a_{n+1}\right|+\left|a_{n+2}\right|+\cdots<\sum_{k=0}^{\infty}\left|a_{k}\right|=\|f\|<\infty
$$


for each $n \in \mathbf{N}$ we obtain

$$
\|g\|=\sum_{n=0}^{\infty}\left|c_{n}\right|<\frac{\|f\|}{1-\left|z_{0}\right|}<\infty
$$

We show, moreover, that $g \in I$. By hypothesis (1) there exists one of the generators of $I$, w.l.o.g. take $f_{1}$, such that $f_{1}\left(z_{0}\right) \neq 0$. Let

$$
h(z)=\left[f_{1}(z)-f_{1}\left(z_{0}\right)\right] /\left(z-z_{0}\right)
$$

We note that $h \in W^{+}$by the same argument as above. The identity $f_{1}\left(z_{0}\right) g(z)=$ $f_{1}(z) g(z)-f(z) h(z)$ now yields $g \in I$.

Continuing this procedure of dividing out the finitely many zeros of $f$ we obtain a function $F \in I$ with no zeros in $\bar{D}$. Using the same constructive argument of Spilker [6] we see that $F$ is invertible in $W^{+}$; thus $I=W^{+}$.

REMARKS. 1. The case $A=W$ is much simpler. As previously mentioned, $f=\sum_{i=1}^{N} \bar{f}_{i} f_{i} \in W$ is, by hypothesis, b.a.f.z. in $\bar{D}$; hence one can use the constructive argument of D. J. Newman [5] to show that $f$ is invertible in $W$.

2. In the case of the algebras $A(\bar{D}), A^{n}(\bar{D})$ one has to note that in general the partial sums of the Taylor series of a function $f$ do not converge to $f$. Therefore the definition of $P_{i}$ in the proof has to be modified. Since the function $f_{r}(z)=f(r z)$ converges to $f$ for $r \rightarrow 1$, one may take for the $P_{i}$ appropriate partial sums of $f_{i}(r z)$. (4) in this case is trivial.

3. Let $f \in W^{+}$. If $B$ is a finite Blaschke product with zeros $z_{1}, \ldots, z_{m}$ (repeated according to multiplicity) and $f / B$ is analytic in $D$, then our proof shows $f / B \in$ $W^{+}$and

$$
\left\|\frac{f}{B}\right\| \leqslant\|f\| \prod_{K=1}^{m} \frac{1+\left|z_{K}\right|}{1-\left|z_{K}\right|} .
$$

4. There are a number of other less well-known algebras in the literature in which our proof works; for example, in the small Lipschitz algebras of analytic functions (see [3] and [4]),

$$
\lambda_{\alpha}:=\left\{f \in A(\bar{D}): \lim _{|z| \rightarrow 1}(1-|z|)^{1-\alpha}\left|f^{\prime}(z)\right|=0\right\}, \quad 0<\alpha<1,
$$

with the norm

$$
\|f\|_{\alpha}=\|f\|_{\infty}+\sup \left\{(1-|z|)^{1-\alpha}\left|f^{\prime}(z)\right|: z \in D\right\} .
$$

5. In our arguments in the proof it was not essential that the algebras are complete or complex, etc., therefore our proof can be used in more general situations.

\section{REFERENCES}

1. L. Carleson, On bounded analytic functions and closure problems, Ark. Mat. 2 (1954), 283-291.

2. P. J. Cohen, A note on constructive methods in Banach algebras, Proc. Amer. Math. Soc. 12 (1961), 159-163.

3. V. I. Gavrilov and G. D. Levšina, Linear functionals on Lipschitz spaces of holomorphic functions in the unit disc, Dokl. Akad. Nauk SSSR 239 (1978), 30-33 = Soviet Math. Dokl. 19 (1978), 259-262. 
4. A. L. Matheson, Closed ideals in rings of analytic functions satisfying a Lipschitz condition, Lecture Notes in Math., vol. 604, Springer-Verlag, Berlin and New York, 1977, pp. 67-75.

5. D. J. Newman, A simple proof of Wiener's 1/f theorem, Proc. Amer. Math. Soc. 48 (1975), 264-265.

6. J. Spilker, A simple proof of an analogue of Wiener's 1/f theorem, Arch. Math. (Basel) 32 (1979), 265-266.

Mathematisches InStitut der Universttät, D-6300 Giessen, Federal Republic of Germany

Current address: Mathematisches Institut I der Universität, D-7500 Karlsruhe, Federal Republic of Germany 